\title{
Immigration et entreprenariat
}

Les Philippins à Paris

\section{Asuncion Flot-Fresnoza et Antoine Pécoud}

\section{(2) OpenEdition}

Journals

Édition électronique

URL : https://journals.openedition.org/remi/4187

DOI : 10.4000/remi.4187

ISSN : $1777-5418$

Éditeur

Université de Poitiers

\section{Édition imprimée}

Date de publication : 1 octobre 2007

Pagination : 199-216

ISBN : 978-2-911627-46-6

ISSN : 0765-0752

Référence électronique

Asuncion Flot-Fresnoza et Antoine Pécoud, «Immigration et entreprenariat », Revue européenne des migrations internationales [En ligne], vol. 23 - $\mathrm{n}^{\circ} 2$ | 2007, mis en ligne le 01 octobre 2010, consulté le 14 avril 2022. URL : http://journals.openedition.org/remi/4187 ; DOI : https://doi.org/10.4000/remi.4187

Ce document a été généré automatiquement le 14 avril 2022.

(c) Université de Poitiers 


\title{
Immigration et entreprenariat
}

\author{
Les Philippins à Paris
}

\author{
Asuncion Flot-Fresnoza et Antoine Pécoud
}

1 Les Philippines sont l'un des plus importants pays d'émigration au monde. On estime que plus de huit millions de Philippins, sur une population d'environ 90 millions, vivent à l'étranger (Commission on Filipinos Overseas, 2005). Les migrants philippins sont fortement représentés parmi la main-d'œuvre temporaire au Moyen-Orient et parmi les marins, tandis que des millions de migrants permanents se sont établis aux ÉtatsUnis (Asis, 2006). Ce sont les femmes qui occupent une place centrale dans l'émigration philippine. Très visibles dans les secteurs des services aux particuliers et de l'industrie du divertissement et du sexe, elles travaillent également dans les hôpitaux, les usines et comme vendeuses, notamment au Moyen-Orient ou en Italie (Campani, 1993 ; Jackson et al., 1999).

2 Malgré l'importance de cette émigration, il existe peu de cas connus d'entreprenariat philippin et les activités commerciales sont sous-représentées parmi les secteurs d'activité des Philippins. Cependant, on observe au cours des vingt dernières années une diversification de leurs modes d'insertion socio-économique dans les pays de destination, et l'on assiste à l'émergence de commerces en Europe et en Amérique du Nord. Cette évolution remet partiellement en cause la figure stéréotypée du domestique philippin et conduit à des reconfigurations au sein de la population. Les Philippins qui quittent le secteur des travaux domestiques et s'engagent dans le commerce offrent ainsi l'opportunité d'examiner des aspects inexplorés de leur émigration. En France, pays vers lequel cette immigration est récente et encore peu importante, l'entreprenariat philippin est en pleine transformation quantitative et qualitative, à l'image de la population originaire de ce pays.

3 Les recherches sur les commerces immigrés traitent généralement des populations numériquement importantes, visibles et bien établies. C'est le cas en France des populations d'origine chinoise, maghrébine ou turque sur lesquelles porte l'essentiel des études disponibles (Guillon et Taboada-Leonetti, 1986; Ma Mung et Lacroix, 2003; Ma Mung et Simon, 1990 ; Péraldi, 2002 ; Raulin, 2000). Les activités commerciales de groupes moins importants restent mal connues ; l'entreprenariat philippin a fait l'objet 
de quelques études de cas aux Pays-Bas (Maas, 2002) et aux États-Unis (Fawcett et Gardner, 1994), mais pas encore en France. S'il est logique que les groupes importants attirent l'attention, l'étude d'économies immigrées moins développées et plus récentes permet non seulement d'appréhender la diversité de l'entreprenariat immigré, mais aussi de comprendre la manière dont celui-ci voit le jour.

4 Si l'entreprenariat philippin à Paris correspond en partie à un cas relativement classique d'ethnic business, certaines de ses caractéristiques le distinguent cependant d'autres situations plus connues. Géographiquement, il ne se concentre pas dans les quartiers populaires, mais plutôt dans les quartiers bourgeois où nombre de Philippins travaillent comme domestiques. En raison de la sur-représentation des femmes dans ce secteur, les entreprises philippines sont majoritairement tenues par des femmes, contrairement à de nombreux cas connus où ces dernières contribuent de façon informelle aux commerces de leurs maris. De plus, la plupart des commerçants philippins préfèrent embaucher des migrants d'origine nationale différente, et leurs activités commerciales ne fournissent donc que peu d'emplois à leurs compatriotes, notamment aux nouveaux arrivés. Cet entreprenariat se caractérise également par l'importance d'un sous-groupe composé d'homosexuels actifs dans les salons de beauté et de coiffure. Enfin, l'Église catholique joue un rôle important dans son développement.

5 En l'absence d'informations sur les activités commerciales des Philippins en France, cet article vise à fournir une vue d'ensemble de ce phénomène. Après avoir présenté les principales caractéristiques de l'immigration philippine vers la France, il décrit l'insertion de l'entreprenariat philippin dans différents sous-groupes qui structurent cette population, dont les réseaux ne se recoupent que partiellement et qui se basent non seulement sur l'origine nationale, mais également sur l'orientation sexuelle, la classe sociale et l'appartenance régionale et religieuse. Il montre que la réussite commerciale exige simultanément des liens forts avec d'autres segments de la population, notamment les non-immigrés et les migrants d'autres origines, ainsi que des liens transnationaux. Finalement, l'article discute les raisons de la faible représentation des Philippins dans le commerce, ainsi que les perspectives de développement de leurs activités commerciales.

\section{Méthodologie et définition}

6 L'article est issu d'une enquête de terrain menée à Paris de novembre 2004 à avril 2005. Les commerces philippins à Paris n'étant pas répertoriés, nous avons adopté une approche de type "boule de neige », en interrogeant des migrants et différents acteurs (tels que l'ambassade des Philippines et l'Église catholique philippine) sur la connaissance qu'ils avaient de ces commerces. Nous avons ainsi recensé 32 commerçants, parmi lesquels 25 ont accepté d'être interviewés. Ces entretiens, menés dans la langue nationale des Philippines (le Filipino), étaient centrés sur les expériences pré-migratoires des migrants, sur leur situation actuelle et leurs activités commerciales, ainsi que sur leurs projets. Nous avons également eu recours à l'observation participante dans un magasin, un restaurant et devant une Église catholique fréquentée par les Philippins dans le 16ème arrondissement de Paris.

7 Notre définition de "l'entreprenariat philippin » se réfère aux activités commerciales effectuées par tous les individus d'origine philippine qui vivent en France. Étant donné 
le caractère récent de leur immigration, il n'y a pas encore de deuxième génération, du moins pas en âge d'être sur le marché du travail ; nous pouvons ainsi utiliser comme critère le fait d'avoir immigré des Philippines vers l'Europe, quelle que soit la nationalité actuelle du migrant. Cette définition ne suppose aucun caractère spécifique en matière de fonctionnement des commerces; ceux-ci peuvent ainsi présenter une importante diversité quant à leurs employés, clients, produits, etc. Elle inclut également tous les types d'activités commerciales, y compris les activités informelles et à temps partiel ; certains migrants mènent en effet de front un travail rémunéré en tant qu'employé et des activités commerciales en tant que travailleur indépendant.

\section{L'immigration philippine en France}

8 L'immigration philippine vers la France a débuté avec la guerre du Liban en 1975, puis la révolution iranienne de 1979 et la guerre Iran-Irak en 1980, quand des familles aisées de ces pays sont venues se réfugier en France en emmenant leurs domestiques philippins. En 2003, la Commission on Filipinos Overseas (2005) recensait 32011 migrants philippins en France, dont 26121 sans-papiers; la précision de ce chiffre est rendue possible par le fait que tous les Philippins à l'étranger, quelle que soit leur situation administrative, sont invités à s'inscrire à l'ambassade des Philippines de leur pays de résidence, ce que beaucoup d'entre eux font. Si la France attire moins de Philippins que d'autres pays européens comme l'Espagne ou l'Italie, la plupart des observateurs s'accordent sur l'augmentation des migrations des Philippines vers ce pays. Aujourd'hui, la plupart de ces immigrés sont actifs dans le secteur des aides à la personne (femmes de ménages, gardes d'enfant, aide aux personnes âgées) ou employés dans des restaurants, hôtels et magasins. En France, ils se concentrent dans les grandes villes telles que Paris, Bordeaux, Marseille, Lyon et Nice. À Paris, Mozère (2005) constate que ces immigrés occupent le marché d'une "domesticité de haut niveau », employés par des familles riches et souvent étrangères.

9 La population philippine en France s'inscrit dans des modèles migratoires très divers. On peut notamment distinguer les migrants légaux des sans-papiers, même si la frontière entre ces deux catégories est floue car les Philippins peuvent passer de l'une à l'autre. Les migrants en situation régulière sont le plus souvent venus en France, comme étudiants ou par le biais du regroupement familial. De nombreuses domestiques devenues commerçantes ont ainsi fait venir en France leurs maris, et parfois leurs enfants. Les migrants en situation irrégulière sont souvent porteurs d'un visa touristique périmé ; dans les années 1980, il était relativement facile d'obtenir un tel visa à l'ambassade de France à Manille. Aujourd'hui, les contrôles sont devenus plus stricts et les clandestins ont recours à de coûteuses agences de recrutement. Les migrants profitent parfois de la liberté de circulation des personnes dans l'Europe de Schengen, obtenant un visa pour un autre pays d'Europe avant de rejoindre la France. Certains réussissent à régulariser leur statut une fois en France : pendant la campagne de régularisation de 1998 par exemple, 925 Philippins en situation irrégulière en France se sont vus délivrer une carte de séjour (Masson et Balarello, 1997).

10 À l'instar de ce qu'on peut constater aux Pays-Bas (Maas, 2002) et aux États-Unis (Ellis et Wright, 1999), la plupart des commerçants philippins en France ont des diplômes universitaires. Plutôt que de fuir la pauvreté, ils réagissent à l'absence de travail correspondant à leur formation aux Philippines et migrent pour accéder à une mobilité 
sociale dont ils ne peuvent bénéficier dans le pays d'origine. La motivation principale des commerçants est de soutenir financièrement leur famille restée au pays. Et bien que vivant en France, les immigrés se préoccupent fortement de leur statut social aux Philippines: si le capital financier est gagné en France, le capital symbolique est accumulé aux Philippines. Cela est d'autant plus le cas que l'emploi qu'ils occupent est en général sans rapport avec leurs qualifications, même si certains commerçants ont étudié la gestion d'entreprise avant de venir en France. Il faut aussi souligner que même des emplois de faible statut social peuvent exiger des qualifications élevées, comme la maîtrise de l'anglais pour un emploi de domestique.

\section{Les commerces philippins à Paris}

11 Les commerces philippins à Paris se concentrent dans les secteurs des services et de l'alimentation. Nous avons recensé six entreprises de balikbayan box (spécialisées dans la livraison à domicile des colis que les migrants envoient régulièrement à leurs familles), de nombreux services de transfert d'argent à domicile (door-to-door money transfer) et deux salons de beauté fréquentés par des Philippins, ainsi que des coiffeurs qui travaillent le plus souvent dans leur propre domicile. Au moins quatre personnes interviewées travaillent dans le secteur de la peinture et de la rénovation du bâtiment. En ce qui concerne l'alimentation, deux restaurants et des épiceries coexistent avec des services de livraisons à domicile de spécialités culinaires et avec un marché informel de nourriture philippine qui se tient chaque dimanche devant une église du $16 \mathrm{e}$ arrondissement. Outre les services et l'alimentation, les autres commerces répertoriés dans cette étude comprennent un magasin de design intérieur, une agence de voyage, une bijouterie, une agence de placement de domestiques, des commerces de cartes téléphoniques internationales et de souscriptions à la télévision satellite philippine, un service de dépannage informatique et une agence immobilière.

Le développement de ces commerces est à l'évidence lié à l'augmentation de la population philippine en France. À la fin des années 1970 les Philippins n'étaient que quelques dizaines à Paris et le seul commerce était un restaurant à la clientèle essentiellement non-philippine. Selon Ronald, commerçant arrivé en France en 1983 :

"Le marché n'était pas suffisant pour ouvrir un commerce, ce n'était pas une question de capital financier. Lorsque vous êtes philippin, vos clients potentiels sont les Philippins, évidemment. Comme les Philippins étaient peu nombreux à Paris, ouvrir un commerce était impossible ".

13 Le développement des commerces s'est fait à partir des années 1990 suite à l'ouverture, en 1993, du premier magasin par une Philippine et son mari français. L'année suivante, deux entreprises de balikbayan box se sont créées, suivies par d'autres types de commerces visant le «marché protégé» constitué par les Philippins. Comme l'expliquent Aldrich et Waldinger (1990), l'accès à un tel marché exige un certain savoir-faire et la connaissance des goûts de la clientèle, ce qui favorise les commerçants de même origine.

14 La concentration des commerçants philippins dans des secteurs limités est également le résultat de la régulation institutionnelle de ces activités commerciales, qui façonne à la fois les contraintes et les opportunités des commerçants (Rath, 2000). En France, les immigrés non-européens ne peuvent exercer de professions juridiques ou sanitaires, et ne peuvent vendre tabac ou boissons alcoolisées. Depuis 1998, les étrangers non- 
européens qui ne sont pas résidents permanents doivent, pour démarrer une activité industrielle, artisanale ou commerciale, obtenir une carte d'identité portant la mention " commerçant ", dont la délivrance est incertaine. Ce contexte institutionnel restreint substantiellement les activités commerciales des immigrés, avec pour conséquence leur engagement dans des activités commerciales moins régulées, comme la restauration, l'industrie de la construction et du textile, l'épicerie, le commerce de gros et de détail (Ma Mung et Lacroix, 2003).

15 Parmi les 25 commerçants interviewés, cinq se trouvent en situation irrégulière et ne peuvent s'engager que dans des commerces informels. Des activités informelles peuvent également être observées parmi les migrants en situation régulière; les commerces informels représentent en effet souvent une étape préalable pour les commerçants débutants : la moitié des commerçants déclarés ont ainsi commencé de façon informelle et ont attendu que leurs commerces deviennent stables pour les déclarer. Les commerces formels attirent généralement des clients d'origine plus variée que les commerces informels, qui visent surtout les immigrés philippins. De plus, de nombreux immigrés cumulent travail rémunéré et activités commerciales pour accroître leurs revenus :

"Je vends de la nourriture philippine seulement le dimanche... de lundi à vendredi, je travaille comme domestique et je rentre chez moi vers 7 heures ou 8 heures du soir. Le samedi, je garde une personne âgée pendant deux heures, et après je commence à préparer et à cuisiner des nourritures philippines pour les vendre le lendemain devant l'église. " (Lili, vendeuse des spécialités philippines)

Dans certains cas, ces activités commerciales « complémentaires » sont une question de survie; dans d'autres cas, ils représentent une stratégie économique qui permet d'accumuler rapidement du capital, comme le constate Parreñas (2001) parmi les domestiques philippins à Rome. Parmi les 18 commerçants qui sont actuellement engagés à plein-temps dans le commerce, 11 ont commencé par une activité commerciale à temps partiel et n'ont mis fin à leurs autres occupations qu'après avoir atteint un certain niveau de succès et de stabilité financière.

Aujourd'hui, la majorité des commerçants interrogés sont âgés d'une quarantaine d'années (âge moyen 45 ans, âge médian 48 ans). La plupart d'entre eux ont en effet commencé à travailler dans les services aux particuliers, ce qui leur a permis d'économiser de l'argent et d'apprendre le français.

\section{Les réseaux familiaux et sociaux}

Parmi les 25 commerçants philippins interviewés, 17 sont mariés. Près de la moitié de ces mariages sont interethniques; ce sont le plus souvent les femmes qui se marient à l'extérieur de leur groupe, généralement avec des Européens. La famille joue un rôle essentiel dans le développement des commerces philippins à Paris: douze commerçants décrivent leur commerce comme une entreprise familiale, cinq comme un partenariat avec des amis, et huit comme une entreprise individuelle. Pour la plupart des commerçants, l'accès au capital financier est fourni par leur propre famille sous la forme de prêts familiaux; le recours au paluwagan (tontine) n'est observé que dans quelques cas. Seuls trois commerçants ont emprunté de l'argent à des amis extérieurs à leurs familles. Plusieurs commerçants expliquent leur réticence à 
emprunter hors de la famille par le souci d'éviter de contracter une dette morale (utang na loob).

19 La plupart des commerçants comptent sur l'aide non-rémunérée des membres de leur famille, dont le travail est considéré comme faisant partie des obligations familiales; c'est également un impératif commercial car l'embauche d'employés extérieurs à la famille serait trop coûteux. Lorsqu'un commerce est lancé, il arrive souvent que les membres de la famille du commerçant réduisent ou abandonnent leurs activités professionnelles afin de contribuer au développement de l'entreprise. Le besoin de réduire les coûts en dépendant exclusivement des membres de la famille conduit ainsi à des conditions de travail qui ne séparent guère vie familiale et vie professionnelle.

Si la participation des familles est une caractéristique fréquente de l'entreprenariat immigré (Light et Gold, 2000), le rôle dirigeant des femmes dans les commerces philippins est plus inhabituel. Les femmes jouent généralement un rôle central dans les entreprises immigrées, mais plutôt en assistant leur mari (Espiritu, 1999); si elles deviennent elles-mêmes commerçantes, c'est souvent en développant des réseaux nonethniques hors de leur groupe d'origine (Dallalfar, 1994 ; Hillmann, 1999). Au contraire, dans le cas de l'entreprenariat philippin, ce sont souvent les hommes qui aident leurs épouses: par exemple, Mme Flora qui dirige une entreprise de balikbayan box peut compter sur son mari et sur ses deux enfants pour aller chercher les colis chez leurs clients ; de la même façon, les maris des vendeuses de spécialités culinaires les aident à les préparer et participent à la vente les dimanches après-midi devant l'église.

21 Le rôle actif des femmes dans le commerce s'explique non seulement par leur savoirfaire (notamment dans les domaines de la cuisine et de la vente), mais aussi par le fait qu'elles sont arrivées en France avant les hommes et ont donc des réseaux plus étendus. Compte tenu de leur sur-représentation dans l'ensemble de la population philippine et leur haut niveau d'éducation, leur participation active dans le commerce n'est pas étonnante. Cinq commerçantes sont par ailleurs mariées avec des Européens et ont bénéficié du soutien moral et financier de leurs maris pour démarrer leur commerce.

22 Outre la famille, les réseaux sociaux jouent un grand rôle. La plupart des Philippins nouvellement arrivés en France s'efforcent de rencontrer des compatriotes avec qui entrer en relations, un processus dans lequel l'Église et les commerces jouent un rôle important. L'amitié contribue également à la création des commerces : par exemple, $\mathrm{M}$. Noli à son arrivée à Paris a rencontré un kababayan (personne de même origine locale et linguistique) qui l'a embauché dans son entreprise de peinture et de rénovation de bâtiments. Après quelques mois d'apprentissage, M. Noli a décidé d'établir sa propre entreprise avec l'accord de cet ami. En remerciement de l'aide qu'il a reçu, M. Noli partage parfois ses contrats avec cet ami et ex-employeur :
"Son nom est très populaire, c'est pour ça que je l'ai contacté et l'ai rencontré. Je lui ai dit que je voudrais apprendre la peinture en bâtiment, mais il m'a donné une tâche moins difficile et moins sale, parce qu'en fait j'étais chauffeur à ce moment-là, portant manteau et cravate. Je lui ai dit, aucun traitement spécial. Après une année, j'ai décidé d'aller solo et faire ma propre carte de visite [...] J'ai une grande dette morale vers lui. S'il y a un grand projet, il me dit "prends-le et je te soutiendrai". » (M. Noli, propriétaire d'une entreprise de peinture et de rénovation de bâtiment)

Les relations amicales caractérisent également la relation entre les commerçants et leurs clients. Les vendeurs de spécialités culinaires devant l'église retrouvent les mêmes clients tous les dimanches et développent avec eux des liens étroits, comme le 
montrent les conversations, plaisanteries et rires entendus après la messe. Les commerçants cherchent ainsi à se constituer une clientèle fidèle afin d'assurer la stabilité financière de leurs entreprises. Les réseaux basés sur l'amitié permettent également la circulation d'informations au sein de la population philippine. Le rôle joué par ces réseaux en fournissant de la main-d'œuvre, des informations économiques et diverses formes d'assistance est une caractéristique fréquente de l'entreprenariat immigré (Light et al., 1993).

\section{Les réseaux basés sur l'orientation sexuelle et la classe sociale} jouent un rôle important dans le développement de l'entreprenariat philippin à Paris. Les différences de classes sociales sont très perceptibles au sein de la population et résistent à l'expérience migratoire et à la mobilité sociale qu'elle peut générer. Les immigrés qui travaillent comme domestiques occupent plus ou moins le même statut social, ce qui crée une solidarité de classe. Parmi les 25 commerçants interviewés, 11 étaient initialement domestiques; ils se connaissent depuis longtemps et maintiennent des liens après être devenus commerçants, constituant ainsi un réseau basé sur leur passé partagé. En revanche, les Philippins qui n'ont jamais été domestiques n'appartiennent pas à ce groupe et interagissent très peu (aussi bien commercialement que personnellement) avec leurs compatriotes d'origine plus modeste. Leurs commerces visent généralement une clientèle française et attirent peu de clients co-ethniques.

Ce clivage au sein de la population philippine illustre la résistance des rapports de classe à la réussite commerciale. Dans le cas des ex-domestiques devenus commerçants, l'entreprenariat peut améliorer leur statut, à la fois en France (dans la mesure où ils sortent de la condition de domestique) et aux Philippines (où leur pouvoir d'achat augmente). Pourtant, ce succès n'entraîne pas de prestige social aux yeux de leurs compatriotes issus de classes sociales plus élevées, car le "stigmate " lié à leur passé perdure. Ceci invite à nuancer l'impact de l'entreprenariat sur la mobilité sociale : le statut d'entrepreneur, quelle que soit sa précarité, est en effet souvent perçu comme permettant aux immigrés d'accéder à la petite bourgeoisie commerçante (Lessinger, 2003). Dans le cas de l'économie philippine, le commerce accroit certes le revenu mais n'affecte guère le statut social, soulignant ainsi le poids du passé dans l'élaboration des identités sociales. Il est encore trop tôt pour savoir si ces clivages persisteront au sein de la deuxième génération d'immigrés philippins.

Parmi les commerçants interviewés, six (cinq hommes et une femme) se déclarent homosexuels. Les premiers Philippins homosexuels sont arrivés à Paris entre la fin des années soixante-dix et le début des années quatre-vingt et sont maintenant installés en France de manière stable, tant professionnellement que juridiquement. Ce groupe prodigue aujourd'hui conseils et assistance aux Philippins homosexuels arrivés récemment à Paris et qui se trouvent majoritairement en situation irrégulière. Des sans-papiers travaillent comme coiffeurs et reçoivent leurs clients dans leurs propres appartements pour un prix très inférieur à un salon de coiffure français, attirant ainsi une clientèle de domestiques et gardes d'enfant philippins. Par ailleurs, les salons de beauté et de coiffure représentent une niche commerciale occupée de façon exclusive

Revue européenne des migrations internationales, vol. 23 - n² 2007 
par des migrants homosexuels. Les liens sociaux sont forts entre homosexuels philippins et se traduisent par la formation d'associations, qui organisent des activités de loisirs comme des compétitions sportives et des concours de beauté ; les autres immigrés sont largement exclus de ce sous-groupe. Une solidarité similaire est observée par Maas (2004) parmi les Philippins homosexuels vivant aux Pays-Bas. À part cette étude, on ne trouve aucune mention d'un tel phénomène dans la littérature sur l'entreprenariat immigré: l'existence d'un sous-groupe composé d'entrepreneurs homosexuels semble donc être une particularité de l'entreprenariat philippin.

\section{L'origine nationale et l'ethnicité interne}

Comme l'illustrent les divisions de classe, de genre et d'orientations sexuelles, parler d'un entreprenariat "philippin» surestime l'homogénéité de ces activités commerciales au détriment de leur diversité interne. L'existence de sous-groupes au sein de la population, qui se chevauchent sans jamais se recouper entièrement, est également mise en évidence par le rôle de l'appartenance régionale. Parmi les vingtcinq commerçants interviewés, dix-huit sont originaires de Luzon, cinq de Mindanao, et un des Visayas. Les Philippins de Paris demandent systématiquement aux nouveaux arrivés leur origine régionale ou provinciale, les langues ou dialectes qu'ils parlent et leur religion.

Ces subdivisions soulèvent la question de l'adéquation entre appartenance nationale et réseaux ethniques. Comme le soulignent Light et al. (1992), cette adéquation est problématique: beaucoup de migrants ne s'identifient pas au pays dont ils sont ressortissants, mais plutôt à une de ses composantes-religieuse, culturelle, linguistique ou régionale. Cela est en particulier le cas pour les pays culturellement hétérogènes et pour les primo-arrivants : souvent en effet, les migrants ne développent que tardivement une identification à leur nation d'origine, les appartenances régionales jouant un rôle plus important dans les premiers temps de l'immigration. Light et al. soulignent justement que cette question est largement ignorée dans la littérature, dans laquelle on parle d'économies "cubaine ", "turque » ou "chinoise " comme si ces entités nationales étaient systématiquement les plus pertinentes; ils proposent par conséquent la notion d'ethnicité interne et montrent que cette identification à un sous-groupe peut jouer un rôle aussi important que l'origine nationale: les individus de même appartenance ethnique interne se comprennent mieux et préfèrent soutenir le bien-être économique de leur propre communauté plutôt que celui de l'ensemble de leurs compatriotes.

Les activités commerciales des Philippins illustrent ce phénomène. Elles sont en effet largement structurées autour de leur appartenance ethnique interne, notamment pour le commerce des produits symboliquement importants comme la nourriture. Les immigrés en provenance d'une région spécifique monopolisent souvent la fabrication et la vente de spécialités culinaires régionales comme le tocino (porc sucré) et le chicharon (peau de cochon frite) qui sont la fierté des Kapampangan (migrants originaires de la région de Pampanga). Le savoir-faire et la légitimité des commerçants au niveau de la distribution des produits régionaux contribuent à l'essor de l'entreprenariat: les réseaux qui connectent les partenaires commerciaux, leurs employés et leurs clients sont très souvent basés sur l'appartenance régionale. 


\section{L'importance des réseaux interethniques}

d'interactions interethniques entre immigrés philippins, Français et migrants d'origines ethniques et nationales différentes. Si une des épiceries se fournit auprès d'une entreprise philippine d'import-export basée aux Pays-Bas, la plupart des produits vendus dans les magasins philippins à Paris proviennent par exemple des commerçants chinois de Belleville et de la Porte de Choisy. Aucun entrepreneur philippin en France ne dispose à l'heure actuelle de réseaux suffisamment développés pour importer ses produits directement des Philippines; depuis 1999 cependant, une entreprise chinoise importe des produits philippins qui sont ensuite vendus dans des magasins tenus par des Chinois. Les réseaux chinois ont ainsi commencé à exploiter le marché des produits philippins ; comme l'observe Ma Mung (2000), les circuits économiques chinois sont en effet organisés autour de deux marchés de biens et de services, le marché chinois intracommunautaire et le marché non-chinois extra-communautaire.

Certains commerçants philippins interagissent également avec des commerçants pakistanais et sri-lankais, qui leur fournissent les cartes téléphoniques qu'ils revendent à leurs propres clients. L'agence de placement de domestiques se caractérise aussi par sa nature interethnique: s'occupant au départ exclusivement des Philippins, ces intermédiaires travaillent aujourd'hui avec des domestiques originaires du Brésil, de Malaisie, de Tanzanie et du Ghana :

« En fait, c'est quand nous avions notre premier restaurant. J'y travaillais à plein-temps et il $y$ avait beaucoup de familles qui cherchaient des gouvernantes, des gardes d'enfants et ils sont venus pour nous demander si nous connaissions quelques Philippins. Nous répondions toujours que nous ne connaissions personne, que ce n'était pas notre branche. Finalement, après cinq ans, nous avons décidé de lancer une telle affaire parce que nous pourrions aider des Philippins et en même temps nous gagnerions de l'argent. C'était l'idée. " (Emelda Pablo, propriétaire d'une agence de placement de domestiques immigrées)

Les réseaux interethniques entre Philippins et Français entraînent parfois la création de nouveaux commerces : un commerçant philippin a décidé de créer une entreprise de balikbayan box suite au mécontentement d'une amie philippine quant à la qualité des emballages utilisés par les entreprises existantes, et c'est l'usine où travaillait le mari français de son amie qui devint le fournisseur des boîtes de la nouvelle entreprise. De la même manière, $\mathrm{M}$. Caballa et un ami français ont décidé d'ouvrir un magasin de design d'intérieur suite aux commentaires élogieux suscités par les sacs et accessoires en coquillages qu'il avait ramenés en cadeaux à leurs amis français. Quant à M. Dominguez, il a débuté dans l'électronique multimédia en dépannant un ami français qui l'a présenté par la suite à des personnes qui recherchaient un informaticien et technicien.

\section{Les conflits et la précarité dans l'entreprenariat philippin}

Les études sur les économies immigrées mettent fréquemment l'accent sur les réseaux et la solidarité "ethniques", ce qui conduit parfois à sous-estimer les conflits et tensions (Sanders et Nee, 1987). La compétition entre commerçants philippins est dure à Paris, principalement en raison de la saturation de certains de leurs secteurs 
d'activité. Par conséquent, ces commerçants se trouvent en situation précaire et doivent développer des stratégies variées pour survivre. Par exemple, pas moins de six entreprises de balikbayan box saturent complètement ce marché ; les commerçants ne peuvent ainsi se permettre des contre-performances : tout retard à la livraison des colis ou toute disparition d'articles conduira inévitablement à une perte de clients. Ils doivent par ailleurs réduire leurs coûts d'exploitation, ce qui implique une forte dépendance vis-à-vis de réseaux sociaux et familiaux aux Philippines et en France afin d'assurer un acheminement efficace et bon marché. Les commerçants qui vendent des cartes téléphoniques internationales se trouvent également en situation d'intense compétition, non seulement entre eux mais aussi avec des migrants d'origines différentes, pour la plupart pakistanais et sri lankais.

Une autre stratégie de survie passe par la confidentialité. Les commerçants philippins développent continuellement de nouvelles stratégies commerciales qu'ils veulent garder secrètes pour ne pas être imités. La discrétion est de rigueur même dans les relations amicales, comme par exemple entre les vendeurs de nourriture devant l'église qui cherchent à garder secrètes leurs sources d'approvisionnement. Pour attirer les clients, les propriétaires de magasins adoptent différentes stratégies, comme l'ouverture de leurs magasins tous les jours de l'année, des horaires étendus, des prix bas et des réductions pour les clients réguliers; d'autre part, ils visent une clientèle variée (philippine, française et d'autres origines) en vendant des produits philippins, mais aussi américains, chinois et japonais. La concurrence entre les coiffeurs est moins marquée, car ceux-ci peuvent compter sur une clientèle fidèle basée sur l'appartenance régionale et linguistique et accueillent également des clients français. De la même manière, les restaurants visant une clientèle non-philippine réussissent à éviter d'entrer en compétition. Cette stratégie de survie des commerçants philippins, consistant à cibler à la fois une clientèle co-ethnique et non co-ethnique, apparaît fréquente chez les commerçants immigrés, comme les Turcs à Berlin (Pécoud, 2001) et les Chinois à Paris (Ma Mung et Simon, 1990).

Certains commerçants utilisent également leur appartenance régionale pour légitimer leur présence dans la fabrication et la vente de spécialités culinaires, tandis que d'autres mettent l'accent sur la nature pionnière de leur commerce afin de discréditer leurs concurrents. Ces attitudes sont parfois dues à la jalousie, qui s'observe fréquemment entre commerçants actifs dans le même secteur. Ainsi, une vendeuse de nourriture insiste sur le fait qu'elle était la première dans ce type de commerce à Paris et que les autres n'ont fait que l'imiter par la suite, thèse naturellement réfutée par d'autres commerçants... La saturation du marché de la nourriture pousse ainsi certains commerçants à utiliser n'importe quelle stratégie afin de surmonter la compétition de leurs concurrents.

\section{Les activités transnationales}

La nostalgie du pays d'origine ne conduit pas seulement les Philippins en France à consommer des spécialités de leur pays d'origine, mais également à acheter ou louer des films philippins, à lire des magazines et des journaux, voire à s'équiper d'un récepteur satellite et d'un décodeur afin de recevoir la télévision philippine. L'émergence de cette pratique de consommation s'accompagne du développement de commerces transnationaux (Portes et al., 2002): balikbayan box, vente de cartes 
téléphoniques internationales, transferts de fonds, location de films philippins, installation des récepteurs satellites, activités immobilières.

Le commerce de balikbayan box s'est modernisé ces quinze dernières années avec le recours des commerçants à l'Internet, au téléphone portable et au fax afin de s'adapter à la demande d'une clientèle croissante. Certains d'entre eux ont affilié leur commerce à des entreprises internationales, ce qui leur procure un avantage compétitif et leur permet de pratiquer des prix plus attractifs. Pour la plupart des commerçants de balikbayan box, il est en outre important d'avoir quelqu'un de confiance aux Philippines, généralement un membre de la famille ou du clan familial qui prend soin de leur marchandise et qui supervise la livraison à domicile des colis.

Le marché des cartes téléphoniques internationales et de la livraison d'argent à domicile renforce les commerces informels des Philippins à Paris. Le commerce de cartes téléphoniques internationales a commencé au début des années quatre-vingt-dix afin de satisfaire la demande croissante des immigrés. Aujourd'hui, quatre Philippins pratiquent ce commerce. Afin de maintenir le lien familial, les Philippins envoient également de l'argent tous les mois par des moyens formels, comme les virements bancaires ou postaux, ou informels, tels que la livraison d'argent à domicile. Cette dernière forme de commerce utilise largement les réseaux familiaux afin d'offrir des prix plus attractifs que les moyens formels et une livraison à domicile très rapide, souvent le jour suivant la commande.

On observe également un marché immobilier florissant: des Philippins à Paris deviennent représentants d'entreprises immobilières basées aux Philippines afin de permettre aux immigrés d'acquérir des biens immobiliers dans leur pays d'origine; des publicités à ce sujet sont affichées à la porte des magasins, ainsi qu'à l'ambassade des Philippines. L'émergence de cette forme de commerce explique en partie pourquoi beaucoup de Philippins vivent dans de modestes chambres de bonnes afin d'épargner l'argent nécessaire à l'acquisition d'un lopin de terre ou d'une maison aux Philippines, symboles de mobilité sociale dans la société d'origine.

Les commerces comme l'immobilier, la location de film et la télévision par satellite sont ainsi en relation avec des commerces basés aux Philippines, qui sont déjà bien établis et cherchent à pénétrer le marché des Philippins de l'étranger. En revanche, il existe pour l'instant peu de liens entre commerces philippins en France et ailleurs en Europe. Ce n'est que récemment que quelques commerçants à Paris ont commencé à étendre leurs commerces, nouant des contacts avec d'autres Philippins à l'étranger. Parmi les 25 commerçants interviewés, 18 ont exprimé leur intention de développer leur commerce en France, mais également dans d'autres pays européens où ils sont en contact avec des migrants philippins :

«En fait, il n'y a pas le choix, qu'est-ce que je vais faire ici? Je pense qu'il est plus facile de commencer un commerce d'import-export ici, puisque j'ai déjà le capital. J'irai en Belgique ou à travers l'Europe [...] en fait j'ai déjà rencontré des gens [philippins] dans beaucoup de réunions religieuses, par exemple entre pays européens. J'en ai rencontré de Suisse, des PaysBas, alors nous avons échangé nos cartes de visite." (M. Noli, propriétaire d'une entreprise de peinture et de rénovation de bâtiment)

41 Il reste à voir si ces plans d'expansion seront couronnés de succès. Cependant, ces projets laissent supposer qu'on peut s'attendre à une transnationalisation croissante de l'entreprenariat philippin dans les prochaines années. 


\section{Le rôle de l'Église}

42 À l'exception d'une musulmane, les 25 commerçants interviewés sont chrétiens, majoritairement catholiques. Si, en s'inspirant de Max Weber, on a parfois conféré aux valeurs religieuses un rôle explicatif dans le développement de l'entreprenariat au sein des populations immigrées, très peu d'attention a été accordée au rôle des institutions religieuses dans l'entreprenariat immigré.

43 Pour les Philippins à Paris, l'Aumônerie Philippine (Filipino Chaplaincy) est une institution essentielle qui pacifie et unifie les différents sous-groupes ethniques et stimule le développement de commerces. La messe en l'église Sainte Bernadette, dans le 16ème arrondissement, est le lieu de rencontre le plus important de la population philippine et dépasse ses frontières internes, permettant aux différents sous-groupes de se côtoyer sans conflit; tout le monde utilise alors la langue nationale des Philippines, le Filipino, faisant ainsi l'expérience d'une solidarité nationale plus forte qu'en d'autres circonstances. Plusieurs immigrés considèrent l'Aumônerie comme le " pouls des migrants philippins », car elle offre soutien psychologique, économique et social aux migrants, et notamment aux sans-papiers.

Du point de vue commercial, l'Aumônerie Philippine est un acteur-clé et la messe du dimanche un événement central dans le travail de beaucoup d'entrepreneurs, notamment des vendeurs de nourritures. L'Aumônerie a également joué un rôle dans le développement initial des commerces philippins à Paris: en 1989, une domestique ayant échappé à son employeur saoudien s'est réfugiée dans l'église et le prêtre philippin de l'époque lui a proposé de vendre des spécialités devant l'église pour gagner sa vie. Ce commerce s'est ensuite développé quand d'autres domestiques en situation irrégulière ont décidé de l'imiter. Le prêtre qui les a aidés est depuis lors rentré aux Philippines, mais ses successeurs continuent à tolérer cette forme d'activité économique. En retour, les vendeurs font des dons à l'église sans que cela ne leur soit imposé. Du point de vue du prêtre, ce commerce attire les fidèles et les pousse à participer régulièrement à la messe, même si les vendeurs eux-mêmes sont trop occupés par la préparation de leurs étalages pour y assister.

45 La plupart des commerçants utilisent la messe du dimanche pour faire la promotion de leurs commerces, oralement ou par le biais de messages publicitaires affichés sur un panneau près de la porte de l'église. La concentration d'immigrés philippins en ce lieu crée une sorte de marché de l'emploi: les informations circulent tandis que s'établissent des contacts entre commerçants, clients, employés et partenaires potentiels. Des Français viennent également à l'église ou prennent contact avec le prêtre pour trouver des domestiques tandis que les immigrés au chômage lui demandent de les aider à trouver du travail. L'Aumônerie fonctionne ainsi comme une institution sociale mettant en relation les Philippins entre eux et avec leurs employeurs français. Cette fonction est d'autant plus importante en raison de l'absence d'autres mécanismes de régulation entre entrepreneurs (comme les associations commerciales). Le prêtre et la messe du dimanche jettent ainsi des ponts entre Philippins de toutes origines et classes sociales, ainsi qu'entre Philippins et non-Philippins. 


\section{Perspectives pour le développement d'une économie philippine à Paris}

Le développement de l'entreprenariat philippin est un phénomène nouveau, non seulement en France ou en Europe de l'Ouest, mais également en Amérique du Nord. Malgré le très grand nombre de Philippins qui vivent et travaillent en dehors de leurs pays, on n'observe pas d'économies philippines bien développées dans les pays d'accueil, ce qui soulève plusieurs questions : pourquoi les Philippins sont-ils ainsi sousreprésentés parmi les travailleurs indépendants dans les pays occidentaux? Le développement de leur entreprenariat à Paris est-il un processus marginal qui ne remet pas en cause les modes d'immigration et d'installation des Philippins, ou cet entreprenariat va-t-il se développer en une véritable économie immigrée capable d'influencer durablement leur incorporation socio-économique? Les données présentées ici peuvent nous fournir quelques éléments de réponse.

Les activités commerciales des Philippins à Paris partagent plusieurs caractéristiques avec des cas d'économies immigrées mieux connues: la dépendance vis-à-vis de la famille et des réseaux sociaux, l'accès à un marché ethnique non contesté, le travail acharné, la compétition, la précarité ou le caractère souvent informel. Ces activités commerciales ressemblent également à l'entreprenariat des Philippins aux Pays-Bas, tel que l'a analysé Maas (2002). Cette convergence pourrait témoigner d'un processus de " rattrapage ", qui verrait les Philippins, à la suite de nombreuses autres populations immigrées (Coréens, Vietnamiens, Chinois, Indiens, etc.), commencer à élaborer leurs propres activités commerciales. Cependant, plusieurs autres facteurs contextuels influencent le développement des économies immigrées et rendent la situation plus complexe.

Un premier facteur est d'ordre temporel. Les Philippins sont arrivés récemment en Europe et n'ont pas encore eu le temps de développer leurs propres activités commerciales. De plus, comme le constate Maas (2002) aux Pays-Bas, les immigrés philippins envisagent souvent leur séjour à l'étranger comme temporaire et tendent à maximiser la somme d'argent envoyé à leur famille restée aux Philippines. Cette disposition tend à décourager l'entreprenariat à cause des risques et des investissements de longue durée qu'il implique. Même si Bonacich (1973) considère que l'entreprenariat est lié à une 'sojourner mentality' (par laquelle les migrants qui vivent temporairement dans un pays investissent dans le commerce, travaillent dur et maximisent leurs revenus avant de rentrer dans leur pays d'origine), plusieurs études ont montré que les migrants avaient besoin de s'installer de façon permanente avant de considérer le commerce comme une alternative socio-économique viable (Pécoud, 2005). Dans le cas des Philippins, ceci pourrait être en train de se produire et l'entreprenariat serait alors amené à se développer dans les années à venir. Le nombre de couples franco-philippins impliqués dans le commerce illustre par exemple, la manière dont l'installation définitive en France contribue à la création de commerces.

Cette entrée des Philippins dans le commerce s'effectue cependant dans un environnement saturé. Ils arrivent en effet trop tard pour bénéficier du processus de « succession écologique », par lequel les migrants remplacent les ressortissants du pays d'accueil dans des secteurs économiques peu attractifs. Il se peut qu'ils bénéficient du « jeu de chaises musicales ethniques » (ethnic musical chairs) décrit par Waldinger (1996) en investissant les secteurs délaissés par des migrants précédemment arrivés et qui ont 
bénéficié d'une mobilité sociale ; mais c'est loin d'être certain : comme décrit plus haut, les Philippins sont en compétition avec d'autres nouveaux arrivés qui ont peu de chances de s'élever dans l'échelle sociale, du moins à court terme. De plus, un certain nombre d'observations montrent que les commerçants chinois ont commencé à s'intéresser au marché philippin; étant donné leurs réseaux et leur importance numérique, il se pourrait qu'ils ne laissent que peu d'espace au développement de l'entreprenariat philippin et qu'ils entravent son intégration verticale.

La taille de la population joue également un rôle. Elle est actuellement peu importante, ce qui réduit le marché potentiel sur lequel les commerçants peuvent compter initialement. La question est donc de savoir si l'immigration philippine ira croissant, constituant ainsi une clientèle bienvenue pour les commerçants déjà établis. La demande dans les secteurs du travail domestique et des aides à la personne est forte et l'on peut imaginer que cela favorisera la continuation de l'immigration philippine; cependant, des politiques migratoires restrictives pourraient limiter les opportunités de migration légale, réduisant ainsi la croissance de la population philippine et créant des conditions très précaires dans lesquelles les migrants en situation irrégulière n'auraient ni le revenu, ni le statut nécessaire pour alimenter la croissance de l'entreprenariat.

51 Le mode d'installation géographique des Philippins est à double tranchant. D'une part, on peut se demander si leur dispersion dans différents quartiers de la ville constitue un obstacle : la concentration résidentielle peut en effet faciliter la création des réseaux nécessaires au développement de l'entreprenariat et l'absence d'un "quartier philippin » serait à cet égard préjudiciable. D'un autre côté, cette dispersion permet aux entrepreneurs d'avoir accès à une clientèle plus variée, ce qui constitue un atout (Kaplan, 1998). Par ailleurs, la diversification contemporaine de l'immigration philippine vers la France pourrait être favorable au développement de l'entreprenariat, l'arrivée de migrants actifs dans d'autres secteurs que le travail domestique créant un besoin d'emplois et une motivation possible à la création de commerces.

La culture des migrants est un facteur qui se voit parfois accorder un rôle pour expliquer l'entreprenariat. On pourrait se demander dans quelle mesure la sousreprésentation des Philippins dans le commerce vient réfuter l'argument culturaliste selon lequel l'entreprenariat parmi les immigrés japonais, coréens, chinois ou indiens serait lié à des "valeurs asiatiques ", incluant la forte capacité de travail, l'esprit de sacrifice pour l'avenir, la valeur du crédit, la patience, le stoïcisme, la frugalité ou la solidarité (Woodrum, 1981). Fawcett et Gardner (1994) considèrent que l'histoire des Philippines, caractérisée par une longue subordination aux puissances occidentales (Espagne puis États-Unis), a habitué les Philippins à des statuts subalternes défavorables à une culture de l'entreprenariat; ceci pourrait expliquer à la fois leur propension à prendre des emplois dans le domaine des services à la personne et leur faible création de commerce.

Il a en effet été montré que l'expérience pré-migratoire du commerce joue un rôle-clé dans la propension des immigrés à créer leur entreprise (Boissevain et al., 1990). Il manque à la plupart des commerçants philippins à Paris une telle expérience, ainsi que des qualifications, du savoir-faire et des outils qui pourraient faciliter leurs activités commerciales. Cet obstacle doit cependant être relativisé, car la rupture qu'entraîne le fait de migrer peut, en reconfigurant l'état d'esprit et le contexte social des individus, leur permettre d'innover et de libérer un potentiel commercial latent (Peterson, 1995). 
Les données présentées dans cet article montrent effectivement que les nouvelles conditions de vie des Philippins influencent leur décision de s'engager dans le commerce : même sans expérience commerciale, ils parviennent à créer et gérer des commerces qui, bien que modestes, peuvent contribuer à instaurer un savoir-faire commercial au sein de leur population.

Il faut finalement noter que nombre de ces immigrés sont hautement qualifiés. Ils possèdent les ressources de classe qui sont généralement considérées comme encourageant l'entreprenariat, comme le capital humain issu de leur formation universitaire. Il se pourrait pourtant que ce soit précisément l'existence de ces ressources qui empêchent le développement de l'entreprenariat: les qualifications des Philippins - et notamment leur maîtrise de l'anglais - leur permettent de trouver des emplois rémunérés et les protègent des difficultés socio-économiques (chômage, sousemploi) qui poussent, selon la théorie dite du désavantage, de nombreux migrants à créer leur propre entreprise (Maas, 2002).

\section{Conclusion}

Cet article a cherché à décrire l'émergence de l'entreprenariat philippin à Paris. L'immigration philippine en France est un phénomène récent qui prend des formes nouvelles à mesure que ces migrants ne se limitent plus à des emplois domestiques. Si le commerce devient une alternative possible, l'avenir de l'entreprenariat philippin est cependant incertain et il sera intéressant de voir si la création d'entreprises se présentera comme une alternative socio-économique sérieuse pour les Philippins en Europe. Leur incorporation en France va en effet dépendre d'un large éventail de facteurs liés à la fois aux caractéristiques de leur départ (motivation, capital humain, ressources financières et matérielles, situation légale ou pas) et au contexte du pays d'accueil (activités des populations immigrées pré-existantes, politiques gouvernementales, discrimination).

En dépit de leur modeste importance numérique, les commerçants philippins vont jouer un rôle dans ce processus, notamment en tant que modèles possibles pour les migrants à venir. Bien que relatif, leur succès inspirera peut-être d'autres immigrés, amorçant ainsi un processus capable de s'auto-entretenir. Finalement, une leçon des études disponibles sur les économies immigrées est que leur développement n'a presque jamais été prévu : le sens de l'initiative des immigrés est un facteur essentiel dans leur capacité à modifier leur environnement et à se créer des opportunités. L'analyse des évolutions contemporaines des migrations philippines vers l'Europe est à cet égard une précieuse source d'informations.

\section{BIBLIOGRAPHIE}

ALDRICH Howard E. et WALDINGER Roger (1990) Ethnicity and entrepreneurship, Annual Review of Sociology, 16, pp. 111-135. 
ASIS MARUJA M. B. (2006) The Philippines' culture of migration, Retrieved 27 September 2006 http://www.migrationinformation.org/Profiles/display.cfm?ID=364

BOISSEVAIN Jeremy, BLASCHKE Jochen, GROTENBERG Hanneke, LIGHT Ivan, SWAY Marlene, WALDINGER Roger et WERBNER Pnina (1990) Ethnic entrepreneurs and ethnic strategies, in R. Waldinger, H. Aldrich et R. Ward Éds., Ethnic entrepreneurs: immigrant business in industrial societies, Newbury Park, California, Sage Publications, pp. 131-156.

BONACICH Edna (1973) A theory of middleman minorities, American Sociological Review, 38 (5), pp. 583-594.

CAMPANI Giovanna (1993) Labour markets and family networks: Filipino women in Italy, in H. Rudolp et M. Morokvasic Éds., Bridging states and markets: international migrations in the early 1990s, Berlin, Sigma, pp. 191-208.

COMMISSION ON FILIPINOS OVERSEAS (2005) Stock estimate of overseas Filipinos (Inter-agency report) as of December 2004, Retrieved 19 January 2006.

http://www.poea.gov.ph/html/statistics.html

DALLALFAR Arlene (1994) Iranian women as immigrant entrepreneurs, Gender and Society, 8 (4), pp. 541-561.

ELLIS Mark et WRIGHT Richard (1999) The industrial division of labor among immigrants and internal migrants to the Los Angeles economy, InternationalMigrationReview, 33 (1), pp. 26-54.

ESPIRITU Yen Le (1999) Gender and labor in Asian immigrant families, American Behavioral Scientist, 42 (4), pp. 628-647.

FAWCETT James T. et GARDNER Robert W. (1994) Asian immigrant entrepreneurs and nonentrepreneurs: a comparative study of recent Korean and Filipino immigrants, Population \& Environment, 15 (3), pp. 221-238.

GUILLON Michelle et TABOADA-LEONETTI Isabelle (1986) Le triangle de Choisy : un quartier chinois à Paris, Paris, L'Harmattan.

HILLMANN Felicitas (1999) A look at the «hidden side»: Turkish women in Berlin's ethnic labour market, International Journal of Urban and Regional Research, 23 (2), pp. 267-282.

JACKSON Richard T., HUANG Shirlena et YEOH Brenda S. A. (1999) Les migrations internationales des domestiques philippines : contextes et expériences aux Philippines et à Singapour, Revue Européenne des Migrations Internationales, 15 (2), pp. 37-67.

KAPLAN David H. (1998) The spatial structure of urban ethnic economies, UrbanGeography, 19 (6), pp. 489-501.

LESSINGER Johanna (2003) Indian immigrants in the United States. The emergence of a transnational population, in B. Parekh, G. Singh et S. Vertovec Éds., Culture and economy in the Indian diaspora, London, Routledge, pp. 165-182.

LIGHT Ivan, BHACHU Parminder et KARAGEORGIS Stavros (1993) Migration networks and immigrant entrepreneurship, in I. Light et P. Bhachy Éds., Immigration and Entrepreneurship. Culture, Capital and Ethnic Networks, London, Transaction Publishers, pp. 25-49.

LIGHT Ivan et GOLD Steven J. (2000) Ethnic economies, San Diego, Academic Press.

LIGHT Ivan, SABAGH Georges, BOZORGMEHR Mehdi et DER-MARTIROSIAN Claudia (1992) Los Angeles : l'économie ethnique iranienne, Revue Européenne des Migrations Internationales, 8 (1), pp. 155-169. 
MA MUNG Emmanuel (2000) La diaspora chinoise : géographie d'une migration, Paris, Ophrys.

MA MUNG Emmanuel et LACROIX Thomas (2003) France: The narrow path, in R. Kloosterman et J. Rath Éds., Immigrant entrepreneurs. Venturing abroad in the age of globalisation, Oxford \& New York, Berg Publishers \& New York University Press pp. 173-193.

MA MUNG Emmanuel et SIMON Gildas (1990) Commerçants maghrébins et asiatiques en France : agglomération parisienne et villes de l'Est, Paris, Masson.

MAAS Marisha (2002) Filipinos in the Netherlands: why aren't they in business?, Asian Migrant, 15 (1-2), pp. 28-34.

MAAS Marisha (2004) Filipino entrepreneurship in the Netherlands. Male and female business activity compared, Retrieved 24 July 2005 - http://www.ru.nl/gap/papers/gapwp04-08.pdf

MASSON Paul et BALARELLO José (1997) Rapport de la commission d'enquête sur les régularisations d'étrangers en situation irrégulière, Retrieved 5 January 2006.

http://www.senat.fr/rap/197-47021/197-47021.html

MOZERE Liane (2005) Des domestiques philippines à Paris : un marché mondial de la domesticité défini en termes de genre, Migrations Société, 17 (99-100), pp. 217-228.

PARREÑAS Rhacel Salazar (2001) Servants of globalization. Women, migration and domestic work, Stanford University Press.

PECOUD Antoine (2001) The cultural dimension of entrepreneurship in Berlin's Turkish economy, Revue Européenne des Migrations Internationales, 17 (2), pp. 153-168.

PECOUD Antoine (2005) Les enjeux de l'entreprenariat immigré, Revue de l'intégration et de la migration internationale, 6 (3), pp. 377-403.

PERALDI Michel, Éd., (2002) La fin des norias? Réseaux migrants dans les économies marchandes en Méditerranée, Paris, Maisonneuve \& Larose - Maison Méditerranéenne des Sciences de l'Homme.

PETERSON Mark F. (1995) Leading Cuban-American entrepreneurs: the process of developing motives, abilities, and resources, Human Relations, 48 (10), pp. 1193-1215.

PORTES Alejandro, GUARNIZO Luis E. et LANDOLT Patricia (2002) Les entrepreneurs transnationaux : une forme alternative d'adaptation économique des immigrés, in M. Peraldi Éds., La fin des norias? Réseaux migrants dans les économies marchandes en Méditerranée, Paris, Maisonneuve et Larose - Maison Méditerranéenne des Sciences de l'Homme.

RATH Jan, Éd., (2000) Immigrant businesses: the economic, political and social environment, Houndmills, Basingstoke, Macmillan Press.

RAULIN Anne (2000) L'ethnique est quotidien : diasporas, marchés et cultures métropolitaines, Paris, L'Harmattan.

SANDERS Jimy M. et NEE Victor (1987) Limits of ethnic solidarity in the enclave economy, AmericanSociologicalReview, 52 (6), pp. 745-773.

WALDINGER Roger (1996) Still the promised city? African-Americans and new immigrants in postindustrial, New York, Cambridge, MA, Harvard University Press.

WOODRUM Eric (1981) An assessment of Japanese American assimilation, pluralism, and subordination, American Journal of Sociology, 87 (1), pp. 157-169. 


\section{AUTEURS}

\section{ASUNCION FLOT-FRESNOZA}

URMIS, Université Paris 7 - Denis Diderot.

ANTOINE PÉCOUD

URMIS, Université Paris 7 - Denis Diderot et Migrinter, Université de Poitiers. 